\title{
Reduction in motor vehicle collisions following treatment of sleep apnoea with nasal CPAP
}

\author{
C F P George
}

\begin{abstract}
Background-Patients with untreated obstructive sleep apnoea (OSA) have increased motor vehicle collisions (MVCs). When successfully treated, they report improved driving and fewer mishaps, but there are few objective data to confirm this. A study was therefore undertaken to examine actual MVC data in a large group of patients with OSA before and after treatment with continuous positive airway pressure (CPAP) compared with a control group matched for age, sex, and type of driver's licence (commercial or noncommercial).
\end{abstract}

Methods-Two hundred and ten patients of mean (SD) age 52 (11) years, body mass index (BMI) 35.5 (10) $\mathrm{kg} / \mathrm{m}^{2}$, apnoeal hypopnoea index (AHI) 54 (29) events/h were treated with CPAP for at least 3 years. MVC records were obtained from the Ontario Ministry of Transportation (MTO) database for patients and an equal number of randomly selected control drivers. MVC rates were compared for 3 years before and after CPAP therapy for patients and for the corresponding time frames for controls.

Results-Untreated patients with OSA had more MVCs than controls (mean (SD) MVCs/driver/year $0.18(0.29) v 0.06(0.17)$, p<0.001). Following CPAP treatment the number of MVCs/driver/year fell to normal (0.06 (0.17)) while, in controls, the MVC rate was unchanged over time $(0.06(0.17) v$ $0.07(0.18), p=N S)$. Thus, the change in MVCs over time between the groups was very significant (change $=-0.12(95 \% \mathrm{CI}$ -0.17 to -0.06$), p<0.001)$ ). The MVC rate in untreated patients $(n=27)$ remained high over time. Driving exposure was not different following CPAP.

Conclusions-The risk of MVCs due to OSA is removed when patients are treated with CPAP. As such, any restrictions on driving because of OSA could be safely removed after treatment.

(Thorax 2001;56:508-512)

Keywords: sleep apnoea; driving; motor vehicle collisions; continuous positive airway pressure (CPAP)
It is widely recognised that patients with obstructive sleep apnoea (OSA) are at increased risk for motor vehicle accidents. ${ }^{1-9}$ Daytime sleepiness, reduced alertness, and impaired concentration are believed to be important factors in increasing accident rates, although the published data have shown only weak correlations between subjective and/or objective sleepiness. Moreover, the risk of accidents varies from series to series. Nonetheless, simulated driving performance improves with successful treatment of OSA with nasal continuous positive airway pressure (CPAP $)^{10}{ }^{11}$ or uvulopalatopharyngoplasty (UPPP) $)^{12}$ and there are several subjective reports of reduced collisions once patients are treated. ${ }^{14-17}$ Findley et al, using objective collision data, recently reported reduced accidents in 36 patients with OSA treated with CPAP compared with 14 untreated patients. ${ }^{18}$ While these findings are consistent with clinical impression, that study is limited by the small numbers and the lack of a control group. In this study we extend these results in a much larger patient sample with age and sex matched controls.

\section{Methods}

The patients in the study represent a subset of subjects outlined in a previous report ${ }^{9}$ who were treated with nasal CPAP. Patients underwent overnight polysomnography for a variety of diagnoses. Overnight polysomnography included measures of EEG (C1/A2, C4/A1, O2/A1), EOG and submental EMG, bilateral tibialis anterior EMG, expired $\mathrm{CO}_{2}$ (qualitative) or thermocouple at nose and mouth to index airflow, respiration: rib cage and abdominal respiratory inductive plethysmography (Respitrace), oxygen saturation with ear oximetry (Ohmeda 3700). Apnoea was defined as a cessation of airflow for at least 10 seconds accompanied by an arousal and/or oxygen desaturation. Hypopnoea was defined as a $50 \%$ reduction in airflow and/or respiratory effort accompanied by an arousal and/or oxygen desaturation of $\geqslant 4 \%$. Using confirmed OSA cases (AHI $\geqslant 10$ events/h) we determined driving status and collision records using the Ministry of Transportation of Ontario (MTO) provincial database. From this group we reviewed clinic notes to determine which treatment(s) were used for each patients (in our clinic standard treatments are used including nasal 
CPAP, oral appliance, surgery (UPPP), and/or behavioural means such as weight loss, avoidance of alcohol, smoking cessation, etc). Because our clinic serves a large referral area, not all patients are routinely followed in our clinic. Patients with mild sleep apnoea were often treated conservatively and returned to the care of their primary/referring physician to implement behavioural measures or have surgery. For patients with moderate to severe OSA nasal CPAP was recommended and was titrated on a second overnight study in all patients. These patients were then reviewed in the clinic first within 2-3 months of CPAP initiation and at 6-12 month intervals thereafter. Patients for whom CPAP was prescribed and who had attended the clinic for follow up for at least 3 years following institution of treatment were contacted by telephone and by mail. Although some patients had moved and were no longer attending the clinic, we attempted contact in all subjects by telephone and/or through mail questionnaires to determine their clinical status. Subjects were asked about their CPAP usage (number of hours of use per night, number of days used per week), the effects and side effects of CPAP, and whether or not they had used other treatments for OSA. Changes in health status were recorded. In addition, subjects were asked to keep a driving log and answer questions about their current driving habits and driving ability and to compare these with their pretreatment driving status. Subjects were given a small stipend to encourage them to complete the questionnaires and driving logs. If the questionnaires and driving logs were not returned or returned incomplete, a second attempt was made to contact by telephone.

Based on the age, sex, and class of driver's licence (commercial or non-commercial/ private operator) of the patients, a control group was created at random using the Ministry of Transportation of Ontario (MTO) database. Collision records were obtained for the 3 years before and 3 years after CPAP treatment in patients and for the same time frame for each matched control. It was not feasible to perform overnight polysomnography to exclude sleep apnoea in control subjects because of distances and costs (Ontario is the largest province in Canada and control subjects were living in all parts of the province). The MTO database provides information on the number of collisions but not the type (single $v$ multiple vehicles) or time of day. Because collisions in both patients and the general population follow a Poisson distribution, Poisson regression models were used to assess differences in collision rates. ${ }^{19}{ }^{20}$ Non-parametric methods were used to compare driving exposure measures before and after CPAP. The study design was approved by the University of Western Ontario review board for health sciences research involving human subjects.

\section{Results}

STUDY POPULATION

Figure 1 outlines the study population. This was derived from all patients who underwent overnight polysomnography for a variety of

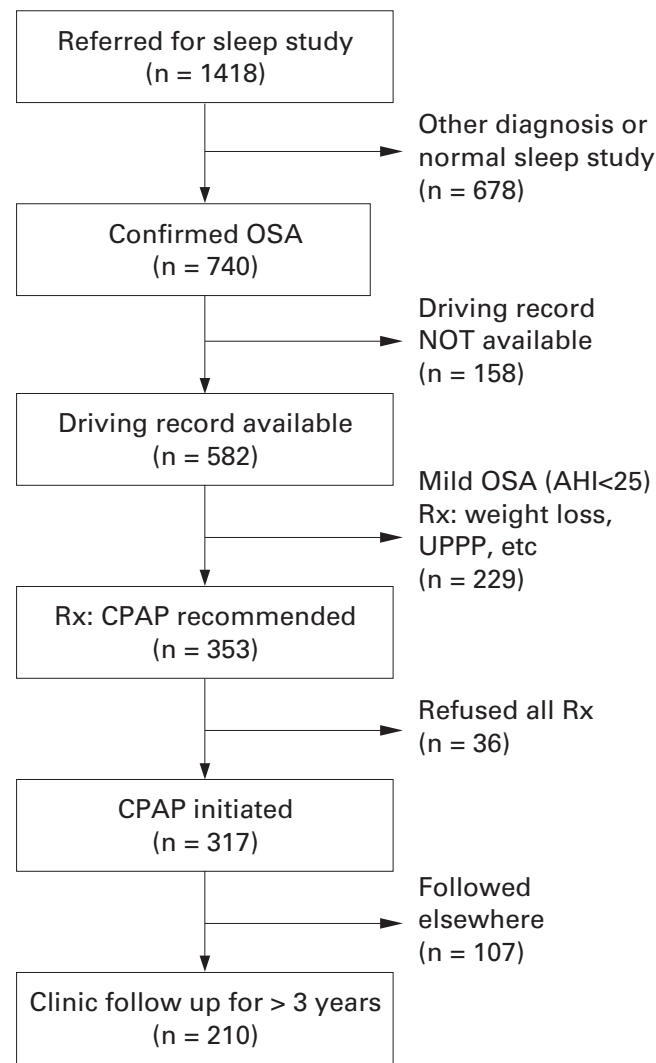

Figure 1 Flow diagram outlining derivation of patient population.

diagnoses between 1 June 1990 and 30 June 1994. Of 740 confirmed cases of OSA (635 men, 95 women), we could confirm driving status and collision records in 582 patients using the MTO provincial drivers' database. Inability to confirm driving status was usually due to change of address (patients had moved or they had left the province) or error in date of birth. The 158 patients for whom collision data were not available (140 men, 18 women; mean (SD) age 50.6 (12.7) years, AHI 41.3 (30.7) events/h) were not different from the 582 for whom collision data were available (524 men, 58 women; mean (SD) age 51.0 (11.9) years, AHI 42.4 (29.3) events/h).

Patients with mild sleep apnoea (AHI <25 events/h) were advised to lose weight and/or discuss surgery and were returned to the care of their referring physician or otolaryngologist. In all patients with AHI $>25$ events/h nasal CPAP therapy was recommended and initiated in the majority. From this group we identified 210 patients (mean (SD) age 52 (11) years, BMI 35.5 (10) $\mathrm{kg} / \mathrm{m}^{2}$, AHI 54 (29) events $/ \mathrm{h}$ ) for whom CPAP was continued for at least 3 years following its institution and who had attended the clinic for follow up for at least that time. The main reason for lack of follow up at our clinic in the other 107 started on CPAP was the distance/time required for travel. During the follow up period weight did not change significantly from baseline in patients with OSA.

CPAP USAGE

Compliance with and treatment effects of nasal CPAP were based on patient self-reports, clinic 

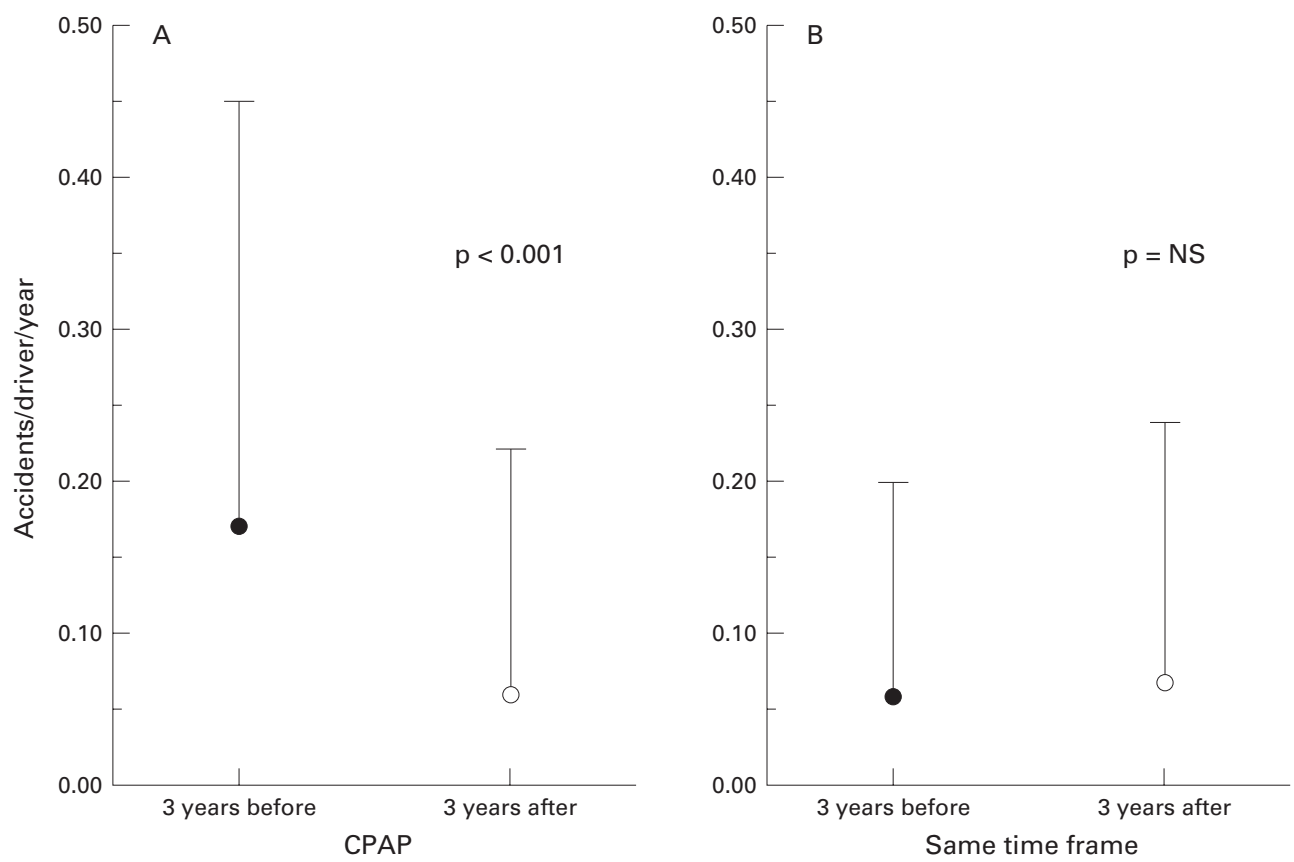

Figure 2 Mean (SD) accident rates for $(A)$ patients with OSA during the 3 years before and after treatment with CPAP and (B) control subjects during the same time frame.

notes and/or follow up questionnaires (via telephone/mail). Of the 210 patients for whom follow up data were available, 182 were current users, 27 were non-users (on no other treatment), five patients had had surgery, and six patients had died. At the time of the current follow up the patients had been on CPAP for 4.2 (1.3) years. Most of the patients (155/182, $85 \%$ ) were well rested with no daytime sleepiness or napping. Nasal congestion or rhinorrhoea was seen in $35 \%$ and $15 \%$ of patients, respectively, but were easily managed with intranasal sprays (anticholinergic and/or topical steroids) or by modifying CPAP circuit humidity. Patients used CPAP on $5.8(0.9)$ days/week for an average of $5.9(0.6)$ hours/day.

MOTOR VEHICLE COLLISIONS

Figure 2 shows the collision rates (number/ subject/year) for patients with OSA during the 3 years before diagnosis and treatment and the 3 years after CPAP treatment, together with the equivalent time frame for controls. Untreated patients had much higher collision rates than controls $(0.18(0.29) v 0.06(0.17), \mathrm{p}<0.001)$ but this rate fell significantly to $0.06(0.17)$ following CPAP treatment, a level not different from that in the control subjects. In contrast, the collision rate was unchanged over time in the controls $(0.06(0.17) v 0.07(0.18), \mathrm{p}=\mathrm{NS})$ such that the change in collision rates between patients with OSA and control subjects was very significant (change $=-0.12,(95 \% \mathrm{CI}$ -0.17 to -0.06$), \mathrm{p}<0.001)$. Collision rates for the 27 patients not using CPAP (and therefore untreated) remained raised over time $(0.15$ (0.26) $v 0.14$ (0.23), $\mathrm{p}=\mathrm{NS})$.

Figure 3 shows the number of collisions in patients before and after CPAP treatment and for control subjects during the same time frame. Single collisions were reduced by nearly $50 \%$ while multiple collisions were reduced even further. However, it is important to notice that most of the patients with OSA did not have any collisions. There was no change in collisions over time in the control group.

\section{DRIVING EXPOSURE}

More than half the patients commuted to work each day before and after treatment and overall there was no change in driving exposure. Based on their driving logs, patients drove 490 (325) $\mathrm{km}$ per week before treatment and 483 (366) $\mathrm{km}$ per week after treatment $(\mathrm{p}=\mathrm{NS}$ ). Nearly equal numbers reported more or less driving following treatment. The patient estimated annual driving exposure was 22700 (16 500) $\mathrm{km}$ which is about double the provincial average of $10850 \mathrm{~km} .^{21}$

\section{Discussion}

Motor vehicle collisions are more frequent in patients with OSA than in matched controls when patients are untreated and this has been reported repeatedly by us and by others. ${ }^{1-9}$ That there should be a similar increase in this group of patients should come as no surprise, particularly since these 210 patients are drawn from a previous report where motor vehicle collisions in patients were increased. ${ }^{9}$ However, these data clearly extend previous literature by demonstrating that collision rates decrease to a rate similar to the general driving population in patients with OSA following successful treatment with CPAP. While Findley et $a l^{18}$ recently reported reduced collisions in a smaller group of patients with OSA treated with CPAP, their study lacked a control group (they compared 36 treated with 14 untreated patients during the same time frame). In this study, not only did we have a much larger patient group but we had matched controls from the general driving population. 

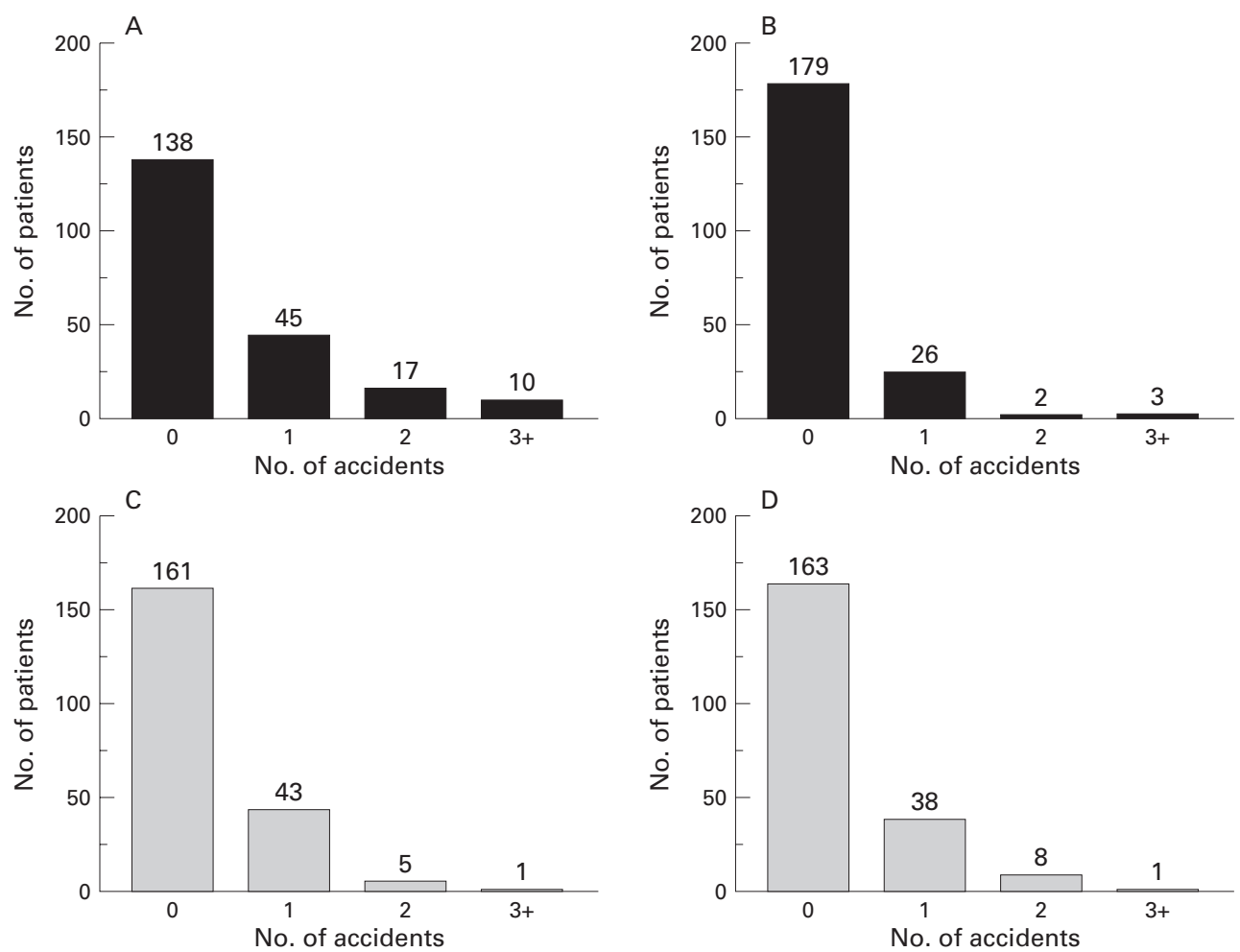

Figure 3 Distribution of accidents in patients with $\operatorname{OSA}(A)$ during the 3 years before and $(B)$ during the 3 years after treatment with $C P A P$, and in controls during the same time frame $(C, D)$.

There are some limitations to this study, the main one being the lack of clinical, polysomnographic, or driving exposure data on the control subjects. As the subjects were randomly selected from the provincial database, it was not possible to perform detailed examinations on these subjects. Moreover, to preserve confidentiality we were not given addresses for these subjects so they could not be contacted to complete driving logs. If, by some chance, all control subjects had little or no driving exposure, then the difference in collision rates between untreated patients with OSA and control subjects, when normalised for driving exposure, would disappear. However, we think this is most unlikely given the random selection of the control subjects. Even in this unlikely scenario, this would not alter our major finding that collisions decrease in treated patients where there was no change in driving exposure.

Nasal CPAP is the treatment of choice for moderate to severe sleep apnoea. Compliance with CPAP was assessed by direct questioning, telephone, and/or mailed questionnaires. While there are inherent sources of bias in these techniques, in clinical practice the physician has little other than the patient's history on which to rely. Assessing CPAP compliance by covert monitoring or newer smart card technologies provides a more accurate assessment of treatment compliance. While this information would have been interesting to know, these technologies were not routinely available during the course of this study. Although it could be argued that this compliance rate is unrealistically high, we think this is unlikely given the magnitude of the improvement in collision rates with treatment. On the other hand, there was no change in collision rates in the control group which, given the high prevalence of sleep apnoea in the general population, would have contained a number of subjects with undiagnosed and untreated OSA.

Another important, but often overlooked, result of this and other studies is the fact that not all patients with sleep apnoea actually have collisions. In this study more than half of the patients never had any collisions either before or after treatment. Still, sleep apnoea is but one of many factors important in motor vehicle mishaps and treatment with CPAP cannot be expected to eliminate collisions completely. The results are consistent with the notion showing that a small percentage of patients had no collisions before treatment but only had mishaps following treatment.

The number of collisions in our patients seems low when expressed as number of collisions per driver per year. However, using the patient based estimates of driving exposure, the number of accidents per 1 million kilometres travelled in the patient group is 2.72 which is almost identical to the provincial average of $2.7 .^{21}$

While OSA patients as a group continue to have increased risk of driving, no studies have yet been able to determine which individuals are at risk. For clinical purposes, however, it is somewhat reassuring that more than half the patients with OSA may never have a collision. Still, if they are at risk, treatment with CPAP will remove the risk due to sleep apnoea. Such results should be of great value to practising physicians who must make assessments about ability to drive, particularly in jurisdictions 
where decisions about ability to drive are influenced by the presence of sleep apnoea.

In summary, we have shown that, using objective driving records, patients with untreated sleep apnoea have increased motor vehicle crashes. This rate decreases to normal once patients are treated with nasal CPAP. While the risk of a collision in the sleep apnoea population is increased, not all patients actually have collisions. Predicting an individual patient's crash risk requires further study.

Funded in part by grants from Ontario Ministry of Transportation and the Medical Research Council of Canada.

1 George CF, Nickerson P, Hanly P, et al. Sleep apnoea patients have more automobile accidents. Lancet 1987;i:447.

2 Findley LJ, Unverzagt ME, Suratt PM. Automobile accidents involving patients with obstructive sleep apnoea. Am Rev Respir Dis 1988;138:337-40.

3 Aldrich MS. Automobile accidents in patients with sleep disorders. Sleep 1989;12:487-94.

4 Haraldsson, P-O, Carenfelt C, Diderichsen F, et al. Clinical symptoms of sleep apnoea syndrome and automobile accidents. ORL 7 Otorhinolaryngol 1990;52:57-62

5 Wu H, Yan-Go F. Self reported automobile accidents involving patients with obstructive sleep apnoea. Neurology 1996;46:1254-7.

6 Young T, Blustein J, Finn L, et al. Sleep-disordered breathing and motor vehicle accidents in a population-based ing and motor vehicle accidents in a population-b

7 Barbé F, Pericás J, Munoz A, et al. Automobile accidents in patients with sleep apnoea syndrome: an epidemiological patients with sleep apnoea syndrome: an epidemiological
and mechanistic study. Am $\mathcal{F}$ Respir Crit Care Med 1998;158:18-22.

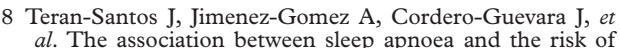
al. The association between sleep apnoea and the
traffic accidents. $N$ Engl f Med 1999;340:847-51.

9 George CFP, Smiley A. Sleep apnoea and automobile accidents. Sleep 1999;22:790-5.

10 George CFP, Boudreau AC, Smiley S. Effect of nasal CPAP on simulated driving performance in patients with obstructive sleep apnoea. Thorax 1997;52:648-53.

11 Hack M, Davies RJO, Mullins R, et al. Randomised prospective parallel trial of therapeutic versus subtherapeutic nasal continuous positive airway pressure on simulated steering performance in patients with obstructive sleep apnoea. Thorax 2000;55:224-31.

12 Haraldsson P, Carenfelt C, Persson HE, et al. Simulated long-term driving performance before and after uvulopalatopharyngoplasty. ORL 7 Otorhinolaryngol 1991:53: 106-10.

13 Haraldsson P-O, Carenfelt C, Lysdahl M, et al. Long term effect of uvulopalatopharyngoplasty on driving performance. Arch Otolaryngol Head Neck Surg 1995;121:90-4.

14 Krieger J, Meslier N, Lebrun T, et al. Accidents in obstructive sleep apnoea patients treated with nasal continuous tive sleep apnoea patients treated with nasal co
positive airway pressure. Chest 1997;112:1561-6.

positive airway pressure. Chest $1997 ; 112: 1561-6$.
15 Minemura H, Akashiba T, Yamamoto H, et al. Traffic acciMinemura $\mathrm{H}$, Akashiba $\mathrm{T}$, Yamamoto $\mathrm{H}$, et al. Traffic acci-
dents in obstructive sleep apnoea patients and effect of nasal CPAP treatment. Fpn F Thorac Med 1993;31:1103-8.

16 Engleman $\mathrm{H}$, Asgari-Jirhandeh $\mathrm{N}$, McLeod A, et al. Self-reported use of CPAP and benefits of CPAP therapy. Chest 1996;109:1470-6.

17 Cassel W, Ploch T, Becker C, et al. Risk of traffic accidents in patients in sleep-disordered breathing: reduction with nasal CPAP. Eur Respir F 1996;9:2606-11.

18 Findley L, Smith C, Hooper J, et al. Treatment with nasal CPAP decreases automobile accidents in patients with sleep apnoea. Am 7 Respir Crit Care Med 2000;161:857-9.

19 Dionne G, Desjardins D, Laberge-Nadeau C et al. Medical conditions, risk conditions, risk exposure and truck drivers accidents: an analysis with count data regression models. Accid Anal Prev

20 Dionne G, Vanasse C. Automobile insurance ratemaking in the presence of asymmetrical information. F Appl Econometthe presence of asym $1992 ; 7: 149-66$.
rict

21 http://www.mto.gov.on.ca/english/safety/orsar/orsar97/

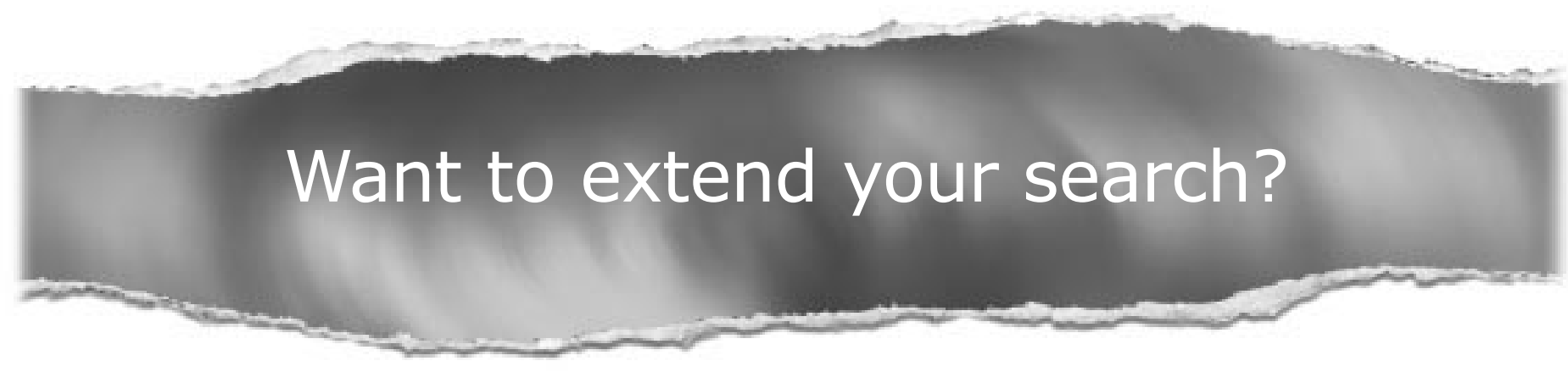

Cross journal searching

If you can't find what you are looking for in Thorax you can extend your search across many of the more than 200 journals available for selection. You can restrict your search to specific subject areas (eg, clinical medicine, basic research), or select specific journals, or search all available titles.

www.thoraxjnl.com 